\title{
A Survey on Coded Cooperative Communication
}

\author{
Chinnu $\mathrm{S}^{1}$, Dr.N Hariharan ${ }^{2}$ \\ ${ }^{1}$ (PG Scholar: Communication Engineering Dept.,ASIET, Kalady, Affiliated to KTU, Kerala, India) \\ ${ }_{2}^{2}$ (Faculty: Electronics and Communication Dept., ASIET, Kalady, Affiliated to KTU, Kerala, India
}

\begin{abstract}
Cooperative communication gains much research attention nowadays to meet the high data rate in cellular networks and wireless ad-hoc networks. User cooperation is promising techniques to improve the performance of wireless networks, where multiple nodes share their resources to achieve transmit diversity. Coded cooperative communication is one of the cooperative system in which cooperation is combined with channel coding there by achieve inherent benefits of spatial diversity and added benefits of channel coding. In coded cooperative communication instead of repeating the codeword each user tries to add some incremental redundancy for its partner. Space time cooperation is an extension to coded cooperative communication in which the second frame itself enjoys the path diversity.
\end{abstract}

Keywords: Cooperative communication, Coded cooperative communication, Space Time cooperation.

\section{Introduction}

Wireless transmission suffers from fading. Transmitting independent copies of the signal can generate diversity which is an effective technique to mitigate the effect of fading. Transmit diversity require multiple transmitting antennas. However most of the wireless devices are limited to one antenna due to their size, cost and hardware complexities. In phase of this limitation Cooperative communication was proposed [1] that enable a single antenna mobile in a multi user environment to share their antennas to create a virtual MIMO system. Cooperative communication is an effective technique to improve the reliability of wireless networks.

Cooperative communication draws from the ideas of using broadcast nature of wireless media. The communicating nodes help each other to implement the communication process in a distributed manner. Three terminal networks is the fundamental unit in user cooperation. The key idea behind the user cooperation is that of resource sharing among multiple nodes in the network [2]. User cooperation improve the system performance such as increase in spectral and power efficiency, network coverage area and reduced outage probability. Figure 1 [1] shows two mobile agents communicating with same destination. In which one antenna mobile cannot individually generate spatial diversity. It may be possible for one mobile to receive the other and forward some version of overheard information along with its own data to the destination. These independent paths can generate spatial diversity. In cooperative communication each user transmits its own data as well as act as a cooperative agent for its partner.

Based on the way how the relay node forwards broadcasted signal to the destination, there are various relaying protocols such as Amplify and Forward (AF), Decode and Forward (DF), Compress and Forward (CF) and Coded Cooperation. In Coded Cooperation instead of repeating the code word each user tries to add some incremental redundancy for its partner. Coded Cooperation uses the same overall rate for coding and transmission; however the coded symbols are rearranged between the two users such that better diversity can attain [3]. Space Time Cooperation was proposed in [4] as an extension to coded cooperation. In space time cooperation the users send both their own as well as their partner's parity bit in the second frame, by partitioning the power of the user in the second frame.

This survey paper formulated by referring reputed journals on cooperative communication and coded cooperative communication. This paper is formulated as follows: section II includes a detailed description of coded cooperation and section III gives a brief description of space time cooperation. Section IV concludes the section.

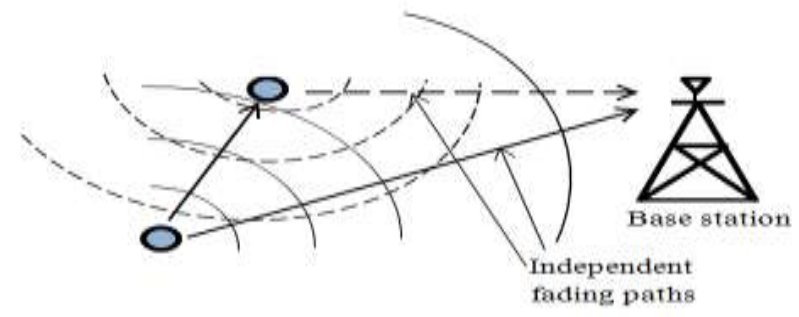

Figure 1: Cooperative Communication 


\section{Relay Protocols}

Co - operative communication uses the broadcast nature of wireless media. In broadcasting period, the neighboring node or the relay node overhear the information and forward it to the destination. At the destination receiver combines the transmitted signal from source and destination and exploits the diversity techniques. Based on how the relay node forwards the broadcasted information to the destination, there are various relay protocols are defined such as Amplify and Forward (AF) and Decode and Forward (DF) and Compress and Forward (CF) and Coded Cooperation (CC).

\subsection{Amplify and Forward}

Amplify and Forward is a simple relay protocol in which the relay node amplify the source transmission and retransmits to the destination. The receiver combines the information received from the source and relay and make a decision. The major limitations associated with amplify and forward relaying is error propagation. i.e., relay node receive a noisy version of source transmission, when the relay amplifies the signal the noise is also get amplified. Another challenges associated with AF relaying is that sampling, amplifying and retransmitting analog values is technologically nontrivial [1].

\subsection{Decode and Forward}

In decode and Forward relaying relay decode the source transmission and re-encode and retransmits to the destination. So DF can limit the error propagation. But decoding errors may occur. When the source relay channel is poor, decoding error may occur, this limits the system performance. Decoding time is also effect the system performance.

\subsection{Compress and Forward}

In Compress and Forward relaying, the relay compress the source transmission and retransmits to the destination. i.e., relay need not to decode and encode the source transmission. So the limitations associated with DF relaying can overcome by this method. The destination can combine the information from source and relay to obtain the original information.

\subsection{Coded Cooperation}

In coded cooperation, channel coding is integrated with cooperation in order to improve the system performance Instead of repeating the code word each user tries to add some incremental redundancy for its partner. The source's data is encoded by any kind of channel coding i.e. block code, convolution code or a combination of both. Then, the data is divided into two parts and each part is transmitted through independent faded channels in the system. In the first transmission, each user sends the first separated part of its own code word as well as tries to decode the other part of separated code word sent from other users. In the second transmission, if each user can decode the data from the other users, it will generate the remaining code word of the other users and transmit the decoded data to the destination. Otherwise, it will transmit the rest of its own code word to the destination.

$\mathrm{AF}, \mathrm{DF}$, and $\mathrm{CF}$ protocols are repetition based relay protocols. The relay node repeats the source transmission. The repetition based relay protocols are not very effective at low SNR values because, the repetition based signaling itself inefficient at low SNR values. Coded cooperative communication shows better performance than other user cooperation methods. Comparison of all the four relay protocols is shown in Table I.

Table I: Comparison Of Various Relay Protocols.

\begin{tabular}{|c|c|c|c|c|}
\hline & $\mathrm{AF}$ & DF & CF & $\mathrm{CC}$ \\
\hline Working & $\begin{array}{l}\text { Relay Amplifies the } \\
\text { signal and retransmit }\end{array}$ & $\begin{array}{l}\text { Relay decode the signal and } \\
\text { retransmit }\end{array}$ & $\begin{array}{l}\text { Relay make an } \\
\text { compressed fom of the } \\
\text { signal and retransmit }\end{array}$ & $\begin{array}{l}\text { Integrate charnel coding } \\
\text { into cooperation }\end{array}$ \\
\hline $\begin{array}{l}\text { Function of the } \\
\text { Relay }\end{array}$ & $\begin{array}{l}\text { Relay act as an analog } \\
\text { repeater }\end{array}$ & $\begin{array}{l}\text { Relays act as digital } \\
\text { regenerative repeaters. }\end{array}$ & $\begin{array}{l}\text { Relays quantize and } \\
\text { compress. }\end{array}$ & $\begin{array}{l}\text { Relay computes parity bits } \\
\text { forits partner. }\end{array}$ \\
\hline Requirement & High S-R channel quality & $\begin{array}{l}\text { Receiver needs CSI between } \\
\text { source and relay. }\end{array}$ & $\begin{array}{lll}\text { Receiver needs } & \text { CSI } \\
\text { between } & \text { source } & \text { and } \\
\text { relay. } & & \\
\end{array}$ & $\begin{array}{l}\text { Relay node must correctly } \\
\text { decode the partner's data. }\end{array}$ \\
\hline Advantage & $\begin{array}{l}\text { Simplest relaying } \\
\text { protocols with low } \\
\text { implementationcost }\end{array}$ & $\begin{array}{l}\text { Minimum error propagation } \\
\text { compared to AFrelaying }\end{array}$ & $\begin{array}{l}\text { Decoding errors can be } \\
\text { overcome. }\end{array}$ & $\begin{array}{l}\text { Flexible and can applied to } \\
\text { verities of channel } \\
\text { conditions. }\end{array}$ \\
\hline Limitation & Noise is also amplified & $\begin{array}{l}\text { When S-R quality is poor, } \\
\text { decoding errors may occur. }\end{array}$ & $\begin{array}{l}\text { Relay need to make an } \\
\text { independent } \\
\text { observation. }\end{array}$ & $\begin{array}{l}\text { Poor perfomance under fast } \\
\text { fading scenario. }\end{array}$ \\
\hline
\end{tabular}




\section{Coded Cooperative Communication}

Coded cooperative communication is one of the cooperative scheme, in which cooperative signaling is integrated with channel coding [3]. Instead of repeating the codeword each user tries to add some incremental redundancy for its partner. The codeword of each user is split into two parts. One part is transmitted by the user and the other part is transmitted by the partner. In addition to coding advantage, coded cooperation also benefited from incremental redundancy. Coded cooperation frame work is illustrated in Figure 2 [2]. In Figure 2 two users communicating with the same destination.

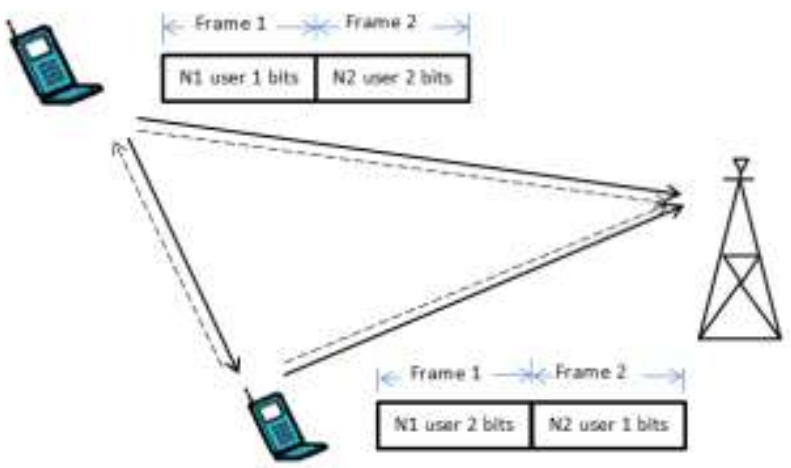

Figure 2: Coded cooperation

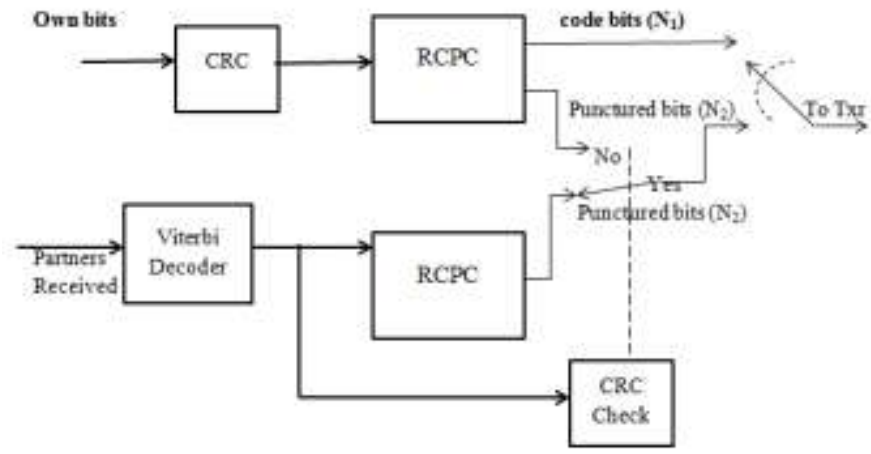

Figure3: Coded cooperation with RCPC codes

User segments their source data and augmented with cyclic redundancy check (CRC) code to get a $\mathrm{K}$ bit block of data. Each block of data is then encoded with forward error correcting code, to obtain an overall rate $\mathrm{R}$ code. i.e., $\mathrm{N}=\mathrm{K} / \mathrm{R}$ bits per block.

The level of cooperation $\alpha$ is defined as

$$
\begin{array}{lllll}
\alpha & = & \mathrm{N}_{2} & /
\end{array}
$$

$\alpha$ defines the percentage of the total bits per each source block that the user transmit for its partner [3].

Various channel coding methods can be used in coded cooperation framework. Entire code can be a block code or a convolutional code or a combination of both. The code can be obtained through puncturing or product code or other form of concatenation. Rate Compatible Punctured Convolutional (RCPC) code based coded cooperation working is shown in Figure 3 [1].

In coded cooperative communication the user act independently in the second frame with no knowledge of whether their own first frame was correctly decoded. Based on that there are four cooperative cases [3]. The four cooperative scenarios are shown in Figure 4 [3].

In Case 1, both users successfully decode each other. So that they can transmit their partner's data in the second frame, and can achieve a fully cooperative scenario. In Case 2, neither user successfully decodes their partner's first frame, the system will automatically reverts to the non-cooperative mode. In Case 3, User 2 successfully decode User 1, but User 1 does not successfully decode User 2. So in the second frame both user transmit User 1's parity bit in the second frame. These two independent copies of Use 1's bits are optimally combined at the destination. In Case 4, User 1 successfully decode User 2 but User 2 does not successfully decode User 1. As a result neither user transmits the second set of code bits for User 1 . 

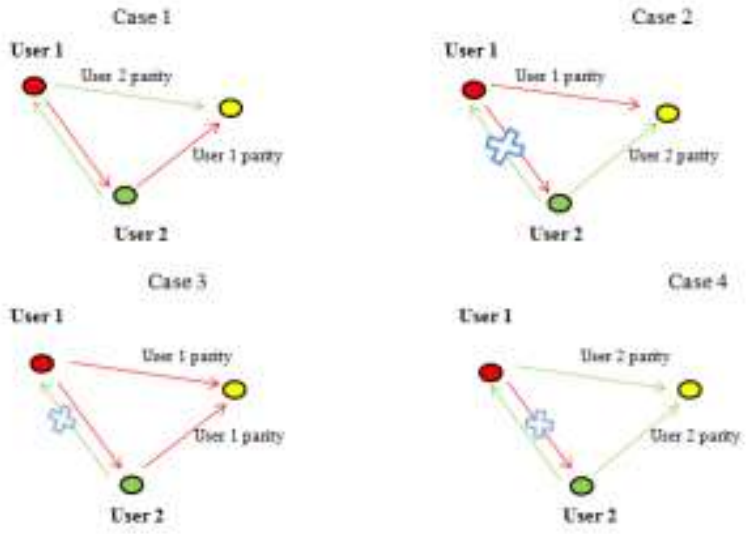

Figure 4: Four cooperative cases

Coded cooperation can be used for a verities of channel conditions. It provide great degree of flexibility by allowing different rate and code partition [1].

\section{Space Time Cooperation}

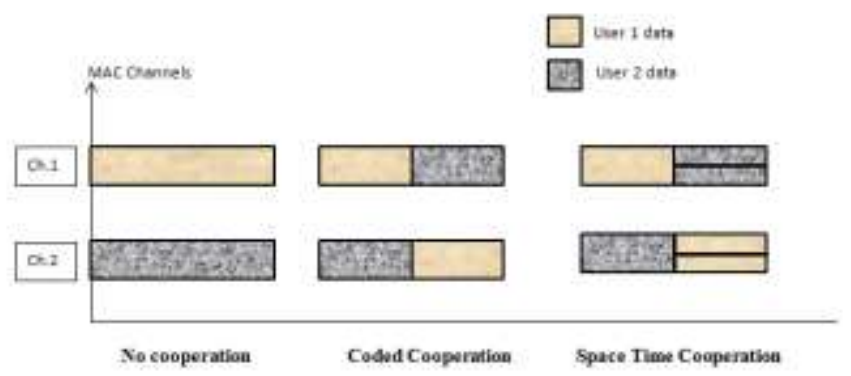

Figure5: Comparison between coded cooperation and space time cooperation

Space Time Cooperation was proposed in [4] as an extension to coded cooperation in which, power of the user is split in the second frame. The users send both their own as well as their partner's parity bit in the second frame. Therefore the second frame itself enjoys the path diversity. Each user makes independent decision on cooperation, based on reception of partner's data. The distinction between coded cooperation and space time cooperation is shown in Figure 5 [3].

Space time cooperation shows better performance than coded cooperation under fast fading scenarios. The reasons are explained in [4] as follows; under fast fading scenarios, first and second frame experiences independent fading. In space time cooperation each user split their power in the second frame and sends both their as well as their partner's data. Thus the second frame itself enjoys the path diversity. In coded cooperation, during case 3 and case 4 only one user is benefited from the transmission of second frame. In fast fading scenario these imbalances can't be tolerated. By sending both users' data in the second frame such imbalances can be tolerated.

Let the encoded block code for User 1 is $Y_{1}$, is divided into two frames $v_{1}$ and $s_{1}$ such that $Y_{1}=\left[v_{1}, s_{1}\right]$. In the frame transmission each user transmit their corresponding $\mathrm{v}_{\mathrm{i}}$ where $\mathrm{i}=1,2$ and tries to decode their partner's $\mathrm{v}_{\mathrm{i}}$ bits. i.e., User 1 transmits its own $\mathrm{v}_{1}$ bits and tries to decode its partner's $\mathrm{v}_{2}$ bits. If the User 1 successfully decode $v_{2}$ User 1 transmits its own $s_{1}$ using User 1's channel and $s_{2}$ using User 2's channel. Otherwise User 1 transmits $s_{1}$ only. In order to maintain same average power User I divide his power in the second frame according to the ratio $\beta_{\mathrm{i}}$. i.e., User I transmits its own $\mathrm{s}_{\mathrm{i}}$ bits with energy $\beta_{\mathrm{i}} \mathrm{E}_{\mathrm{bi}}$ and its partner's bits with energy $\left(1-\beta_{\mathrm{i}}\right) \mathrm{E}_{\mathrm{bi}}[4]$.

Similar to coded cooperation four cooperative cases has to be analyzed. Because user act independently in the second frame. The four cooperative case are as follows [4]. In case 1, both users successfully decode their partner's data, so in the second frame they will transmit their own and their partner's second set. Resulting in a fully cooperative scenario. In case 2, neither users successfully decode their partner's first frame and the system will revert back into non cooperative mode. In case3 User 2 correctly decode User 1 's data but User 1 does not correctly decode User 2's data. As a result User 1 transmits only its own bits in the second frame. User 2 transmit both its own as well as User 1's additional parity bits in the second frame by splitting their power in the second frame. Case 4 is similar to case 3 where role of User 1 and User 2 are reversed. 


\section{Conclusion}

Co-operative communication is a promising solution to meet the high data rate in wireless networks. User cooperation in wireless networks can achieve spatial diversity where the user is equipped with single antenna. Various repetition based relay protocols such as Amplify and Forward (AF), Decode and Forward (DF), Compress and Forward (CF) are widely used in various applications. Repetition based relaying protocols shows a pure performance at low SNR values. In coded cooperative communication cooperation is combined with channel coding and this scheme allows varying the code rate for transmission, i.e., coded cooperation can apply to verities of channel conditions. In fast fading scenario extended version of coded cooperation known as space time cooperation can be used to obtain better performance.

\section{References}

[1] Nosratinia, Aria, Todd E. Hunter, and Ahmadreza Hedayat. "Cooperative communication in wireless networks." IEEE communications Magazine 42.10 (2004): 74-80.

[2] Hunter, Todd E., and Aria Nosratinia. "Performance analysis of coded cooperation diversity." Communications, 2003. ICC'03. IEEE International Conference on. Vol. 4. IEEE, 2003.

[3] Hunter, Todd E., Shahab Sanayei, and Aria Nosratinia. "Outage analysis of coded cooperation." IEEE Transactions on Information Theory 52.2 (2006): 375-391.

[4] Janani, Mohammad, et al. "Coded cooperation in wireless communications: space-time transmission and iterative decoding." IEEE Transactions on Signal Processing 52.2 (2004): 362-371.

[5] Laneman, J. Nicholas, David NC Tse, and Gregory W. Wornell. "Cooperative diversity in wireless networks: Efficient protocols and outage behavior." IEEE Transactions on Information theory 50.12 (2004): 3062-3080.

[6] Hunter, Todd E., and Aria Nosratinia. "Distributed protocols for user cooperation in multi-user wireless networks." Global Telecommunications Conference, 2004. GLOBECOM'04. IEEE. Vol. 6. IEEE, 2004.

[7] Hunter, Todd E., and Aria Nosratinia. "Diversity through coded cooperation." IEEE transactions on wireless communications 5.2 (2006): 283-289.

[8] Bansal, Ankur, and Parul Garg. "An analytical approach to outage analysis and critical cooperation ratio in coded cooperation." Wireless personal communications 69.2 (2013): 721-733.

[9] Fitzek, Frank HP, and Marcos D. Katz. Cooperation in wireless networks: principles and applications. New York: Springer, 2006. 